\title{
Virtues of Using a Double Lumen Tube for Anesthesia in Patients with Endobronchial Tumours Requiring Open Lung Resection or Bronchoplastic Procedures
}

\author{
Assem Adel Moharram* (i), Hany Hasan Elsayed (1) \\ Faculty of Medicine, Ain Shams University, Cairo, Egypt \\ Email: ^A.Adel.Moharram@gmail.com, *assemmoharram1975@gmail.com, hanyhassan77@hotmail.com
}

How to cite this paper: Moharram, A.A. and Elsayed, H.H. (2020) Virtues of Using a Double Lumen Tube for Anesthesia in Patients with Endobronchial Tumours Requiring Open Lung Resection or Bronchoplastic Procedures. Open Journal of Anesthesiology, 10, 1-12.

https://doi.org/10.4236/ojanes.2020.101001

Received: December 8, 2019

Accepted: December 27, 2019

Published: December 30, 2019

Copyright $\odot 2020$ by author(s) and Scientific Research Publishing Inc. This work is licensed under the Creative Commons Attribution International License (CC BY 4.0).

http://creativecommons.org/licenses/by/4.0/

\begin{abstract}
Objective The benefits and risks of using double lumen tube (DLT) in open thoracotomy are not well studied and the relative contraindication for using it in cases of endobronchial tumours is not proven. In this study, we compared our experience with using DLT versus single lumen tube (SLT) for anesthesia in patients requiring an open thoracotomy for resection of endobronchial tumours. Methods A prospective observational study was performed in a single tertiary care university hospital in patients with endobronchial tumours anesthetized with single and double lumen tubes for open thoracotomy procedures over a period from 2010 till 2018. Results One hundred and six patients with endobronchial tumours were studied. There were 76 males. Median age was 32 years (14 - 62). In 96 cases, endobronchial pathology was a typical carcinoid. 58 patients were anesthetized using a DLT and 48 using a SLT. Four cases of near miss from potentially fatal intraoperative tumour migration occurred in the SLT group $(p=0.025)$. There was only one case of mild tumour bleeding from the DLT group and time of insertion was longer (16.2 vs. $4.5 \mathrm{~min} \mathrm{p}<0.001)$ but intraoperative surgeon's satisfaction among the DLT group was $97 \%$ in comparison to only $71 \%$ in cases of SLT insertion $(\mathrm{p}<0.001)$. Conclusions We conclude that it is safe to place a double lumen endo tracheal tube for patients with endobronchial tumours requiring open lobectomies or bronchoplastic procedures. An additional benefit of DLT use is increasing surgical satisfaction by reducing spillage and tidal volume loss during surgical anastomosis of the open airway.
\end{abstract}

*This study was done in the thoracic surgical department of Ain Shams university educational hospital, Cairo, Egypt. 


\section{Keywords}

Double Lumen Tube, Endobronchial Tumor, Thoracotomy

\section{Introduction}

Double-lumen endotracheal tube (DLT) insertion is the most common anesthetic technique to achieve lung separation. The DLT, comprising two parallel lumens, is placed after sizing through the vocal cords into the trachea with a shorter tracheal lumen terminating in the tracheal, and a second, longer lumen extending distally into either the left or right main bronchus. Each lumen has its cuff that is inflated to create the required seal. This allows the anesthetist to ventilate both lungs or the right or left lungs independently [1].

The classic indications for a DLT in achieving single lung ventilation are to confine infections or bleeding to one lung, severe hypoxemia in single lung pathology (e.g. bronchopleural fistulae and major bronchial disruption) and for patients undergoing thoracic surgery procedures to achieve a static field and improve visualization. In video assisted thoracoscopic surgery (VATS), DLT is an absolute indication and failure to achieve single lung ventilation precludes a VATS procedure in most cases [2].

In open thoracotomy cases where patients are considered not suitable for a VATS procedure, a DLT may be used as an adjunct to surgery but is not a must and occasionally some surgeons prefer not to insert a DLT to avoid its potential complications or due to unavailability of an experienced anesthetist or fiberoptic bronchoscopy used to guide its insertion. Additionally, patients with endobronchial tumours may be considered a relative contraindication for insertion of a DLT due to chances of tumour disruption by inadvertent wrong insertion of DLT in the contralateral bronchus [3].

The benefits and risks of using DLT in open thoracotomy are not well studied and the relative contraindication for using it in cases of endobronchial tumours is not proven. In this study, we will compare our experience in a tertiary referral centre with using DLT versus single lumen tube for anesthesia in patients requiring an open thoracotomy for resection of endobronchial tumours via anatomical resections or bronchoplastic procedures.

\section{Patients and Methods}

A prospective observational study was done between 2010 and 2018 for patients requiring lung resection via a thoracotomy for endobronchial tumours in a tertiary referral center. Out of a total of 764 patients who had a lung resection via a thoracotomy procedure, 106 patients had an endobronchial tumour and underwent an anatomical resection or lung sparing procedure by means of a bronchoplastic resection.

Inclusion criteria included all patients aged 16 - 80 years old undergoing lung resection via conventional or sleeve lobectomy due to presence of a lung tumour 
with endobronchial element via a thoracotomy incision. Patients having a thoracoscopic lobectomy or tumour with no endobronchial element were excluded.

All patients were symptomatic and in most cases the symptoms were those of bronchial obstruction: hemoptysis, dyspnea, cough, recurrent infections and fever. No patient presented with carcinoid syndrome. The use of DLT or SLT for anesthesia was based on surgeon's and anesthetist's preference and the practice they felt more comfortable with.

Preoperative evaluation was the same as that for patients with lung cancer. Flexible and/or rigid bronchoscopy was performed in all patients. Biopsy specimens were taken and sent for pathological examination. Preoperative functional evaluation included standard pulmonary function tests.

Time to insert the tube (muscle relaxant to finish tube fixation), complications during tube insertion and any intraoperative complications related to the type of anesthetic tube inserted were recorded.

An intraoperative surgeon satisfaction verbal survey was performed while performing the lung resection procedure to detect possible surgical obstacles associated with SLT in the form of blood spillage and tidal volume loss and with DLT in the form of obscuring the surgical field while performing bronchial anastomosis in bronchoplastic procedures. Follow up was performed until time of patient discharge.

\subsection{Anesthetic Technique}

Pre-anesthetic medication was administered in the form of midazolam $2 \mathrm{mg}$ intravenous in the preoperative room. Standard anesthetic technique for general anesthesia was followed in all patients. Baseline vital parameters were recorded. Anesthesia was induced with propofol $1-2 \mathrm{mg} / \mathrm{kg}$ and fentanyl $2 \mathrm{ug} / \mathrm{kg}$ and 1 $\mathrm{ug} / \mathrm{kg}$ during skin incision after positioning. Tracheal intubation was facilitated by rocuronium $0.5 \mathrm{mg} / \mathrm{kg}$. Anesthesia was maintained by isoflurane in oxygen to achieve a MAC of 1 - 1.2. Patients were monitored by using continuous electrocardiogram, oximetry and end tidal $\mathrm{CO}_{2}$. An arterial catheter was placed for invasive blood pressure monitor. All patients received ondansetron $0.1 \mathrm{mg} / \mathrm{kg}$ to prevent postoperative nausea and vomiting.

Patients were intubated with a Mallinckrodt TM (Coviden, Mansfield) DLT. Left tubes were used except for left pneumonectomy procedures. Tubes were allocated according to height: women $\leq 160 \mathrm{~cm} \mathrm{35F,}>160 \mathrm{~cm} 37 \mathrm{~F}$, men $\leq 170 \mathrm{~cm}$ $39 \mathrm{~F},>170 \mathrm{~cm} 41 \mathrm{~F}$. Distance markings on the tube at the teeth were noted. The bronchial cuff was identified by fiber-optic bronchoscopy (FOB) and moved if necessary, to correct position. Distance mark at teeth was noted again. During insertion, when the double lumen tube tracheal cuff was below the cords, the tube was rotated towards bronchus that is to be cannulated, and the patient's head was turned to opposite side and tube was gently pushed down until resistance is felt. Ventilation is checked once changes have been made between normal ventilation and isolated lung ventilation. If there is any doubt, a fiberoptic bronchoscopy is used to confirm the position of the tube through visualization 
of the bronchial cuff through the tracheal lumen.

\subsection{Surgical Procedure}

Surgical resection was performed through a posterolateral thoracotomy through the bed of the 6th rib. If a classic lobectomy was required, the lobar bronchus was cut and handsewn to achieve a safety margin which would not be feasible with using a surgical stapler for the bronchus. Bronchoplastic procedures were performed whenever the tumor was too close to the bronchial opening or when the bronchial orifice (either lobar or segmental) was involved, in order to avoid unnecessary loss of functional lung tissue and to ensure complete resection of the tumour. A systematic mediastinal lymph node dissection was carried where preoperative diagnosis was not available while nodal sampling in cases of preoperative diagnosis of typical carcinoid pathology in the others. Pathological frozen section examination of the margins of the bronchus was obtained in patients where complete resection was doubtful and when negative margins were confirmed by the pathologist, the bronchoplastic procedure was safely completed. The posterior bronchial anastomosis was performed using continuous suturing (polyglactin or polidioxanon 4/0) while the anterior bronchial wall was anastomosed using interrupted sutures (polyglactin or polidioxanon 3/0) for both full sleeve resections and other bronchoplastic procedures. The suture line was always covered with a pedicled pericardial fat pad or parietal pleura. At the end of the operation, before extubation, the bronchial tree was inspected using a flexible bronchoscope when available to evaluate the suture line, the patency and the alignment of the bronchial anastomosis and to aspirate secretions and blood.

\section{Ethical Considerations}

Ethical committee approval was registered under no 2010/RET-ASU/32 in Ain Shams University (chaired by prof Ayman Shoeb) on $14^{\text {th }}$ of March 2010. Participants were given a fully informed written consent to participate in the study. Participant confidentiality and data security were guaranteed. Participants were able to withdraw from the research process at any time; they were also able to withdraw their data if it was identifiable as theirs and were told when this will no longer be possible. Any expected benefits or potential harm for the research participant was discussed in detail.

\section{Statistical Analysis}

Statistical methods involved in carrying out this study included planning, designing, collecting data, analyzing, drawing meaningful interpretation and reporting of the research findings. The collected data was revised, coded, tabulated and introduced to the PC using Statistical package for Social Science SPSS V20 (SPSS Inc, Chicago, IL). Data was analysed using student paired t test and chi-square test. Data was presented as mean \pm SD and numbers (percentages); as indicated. A p value of less than 0.05 was considered significant. 


\section{Results}

A total of 106 patients were involved in the study over an eight-year period. The median age of the whole group was 32 years $(14-62)$. There were 76 males and 30 females.

In the first group $=58$ patients $(55 \%)$, a double lumen tube was used for anesthesia. The median age was 36 years old (14 - 62). Thirty-nine were males. Typical carcinoid was the most common endobronchial pathology in 52 patients (90\%). Four patients had a diagnosis of atypical carcinoid, one patient with muco-epidermoid tumour and one patient with a squamous cell carcinoma. A bronchoplastic procedure was needed in $53 \%$ of cases while an anatomical lobectomy was performed in $37 \%$ of cases and a pneumonectomy in $10 \%$ of cases. The median time of tube insertion was 16 minutes (3 - 54 minutes) and during insertion one patient (2\%) suffered from significant endobronchial bleeding due to tumour disruption by inadvertent wrong placement of the bronchial tube in the contralateral bronchus. Repeated suction irrigation with saline/adrenaline, washings and partial bronchoscopic compression was enough for the bleeding to cease. No major intraoperative events related to the anesthetic tube occurred and the surgeon's satisfaction with the surgical field was present in 56 out of 58 patients. In both patients with dis-satisfaction, the double lumen tube required multiple repositioning after tube migration (guided intraoperatively by the surgeon) while the airway was open during bronchoplastic procedures which the surgeon felt was a cause of intraoperative delay.

In the second group $=48$ patients $(45 \%)$, a single lumen tube was used for anesthesia. The median age was 34 years old (16 - 62). Thirty-seven were males. Typical carcinoid was the most common endobronchial pathology in 44 patients (92\%). Three patients had a diagnosis of atypical carcinoid and one patient had an endobronchial rhabdomyosarcoma. A bronchoplastic procedure was needed in 26 cases $=54 \%$ of cased while an anatomical lobectomy was performed in $40 \%$ of cases and a pneumonectomy in $6 \%$ of cases. The median time of tube insertion was 4 minutes ( 1 - 13 minutes) and no tumour disruption occurred during tube insertion as care was taken not to advance the tube inadvertently in any major bronchus. Surgeon's satisfaction with the surgical field was present in 34 (71\%) out of 48 patients although surgeons considered this safer for patients. The main cause of dis-satisfaction was continuous interruption of the procedure to avoid loss of tidal volume with open airway and spillage of blood in the contralateral side requiring intermittent suction to avoid attacks of intraoperative hypoxia.

There was no statistical significance in comparing both groups regarding demographics, type of pathology and type of resection done. The DLT had a significantly longer time to tube insertion $(16.2$ vs 5.3 minutes $p<0.001)$ and one event of tumour bleeding from contacting the tube but this was not statistically significant $(\mathrm{p}=0.361)$. On the other hand, four cases of near miss occurred in the SLT due to intraoperative tumour migration $(\mathrm{p}=0.025)$ and surgeon's satisfaction was higher in the DLT group $(97 \%$ vs $71 \% \mathrm{p}<0.001)$. The cost of the 
tubes in the DLT group was $4475 \$$ for the whole group in comparison to only $96 \$$ to the SLT group $(\mathrm{p}<0.001)$.

A comparison of demographics, preoperative and intraoperative events between both groups is shown in Table 1.

\section{Near Miss Events over the Eight-Year Study Period}

There were four patients who suffered a catastrophic intraoperative near miss event in the single lumen tube group.

The first patient was a 29-year-old male who had a typical carcinoid tumour in the left upper lobe and required a left upper lobe sleeve lobectomy. While manipulating the lobe and prior to airway opening, the patient suffered a hypoxic cardiac arrest with sudden increase in ventilatory airway pressure. A contralateral pneumothorax was first suspected, but no air could be aspirated from the contralateral pleural. The patient was immediately transferred to a supine position and a rigid bronchoscopy inserted with the carcinoid found to completely occlude the right main bronchus. The tumour was extracted and the patient was successfully resuscitated, and the procedure completed uneventfully. The patient suffered from a transient period of brain edema but recovered well postoperatively.

The second patient was a 34-year-old male who had a typical carcinoid tumour in the left lower lobe bronchus requiring a left lower lobectomy with hand sewing of the lobar bronchus to achieve a complete resection due to proximity of the tumour from the secondary carina. Just before cutting the bronchus, the patient suffered an attack of severe hypoxia and the anesthetist failed to ventilate the patient. The patient was put in the supine position and a rigid bronchoscopy was used to extract the tumour which was found occluding the bronchus intermedius. The procedure was continued uneventfully, and the patient had an uncomplicated postoperative course.

Table 1. Comparison of preoperative and intraoperative variables between the single and double lumen tube groups.

\begin{tabular}{|c|c|c|c|}
\hline Variable & $\begin{array}{l}\text { Double lumen tube } \\
\text { group }(\mathrm{n}=58)\end{array}$ & $\begin{array}{l}\text { Single lumen tube } \\
\text { group }(n=48)\end{array}$ & $\begin{array}{l}\text { Significance } \\
P \text { value }\end{array}$ \\
\hline Male/female & $39 / 19$ & $37 / 11$ & 0.263 \\
\hline Age & $36(14-59)$ & $34(16-62)$ & 0.187 \\
\hline $\begin{array}{l}\text { Preoperative pathology of } \\
\text { carcinoid }\end{array}$ & $52(90 \%)$ & $44(92 \%)$ & 0.724 \\
\hline $\begin{array}{l}\text { Bronchoplastic procedure } \\
\text { (sleeve resection) }\end{array}$ & $31(53 \%)$ & $26(54 \%)$ & 0.941 \\
\hline $\begin{array}{l}\text { Significant bleeding during } \\
\text { insertion }\end{array}$ & 1 case $(2 \%)$ & None & 0.361 \\
\hline $\begin{array}{l}\text { Median time of tube insertion } \\
(\mathrm{min})\end{array}$ & $16.23 \pm 2.14$ & $4.53 \pm 1.6$ & $<0.001$ \\
\hline $\begin{array}{l}\text { Major intraoperative events } \\
\text { related to the tube }\end{array}$ & None & $4 / 48(8.3 \%)$ & 0.025 \\
\hline $\begin{array}{l}\text { Intraoperative Surgeon's } \\
\text { satisfaction }\end{array}$ & $56 / 58(97 \%)$ & $34 / 48(71 \%)$ & $<0.001$ \\
\hline
\end{tabular}


The third patient was a 45 -year-old male with a rhabdomyosarcoma occluding the right main bronchus and requiring a right pneumonectomy for resection. Due to the extensive tumour bulk, difficult manipulation was performed to expose the hilum and suddenly the patient developed severe hypoxia and bradycardia. The patient was put in supine position and a rigid bronchoscopy was used to extract large pieces of the tumour which were found occluding the left main bronchus. A bronchial clamp was then used to occlude the right main bronchus and prevent this event from recurring intraoperatively. The procedure was continued uneventfully, and the patient had an uncomplicated postoperative course.

The last patient was a 36-year-old female who had a typical carcinoid in the bronchus intermedius requiring a sleeve middle and lower bi-lobectomy. After 30 minutes from starting the procedure, the patient developed a hypoxic cardiac arrest. The index of suspicion was low for tumour migration and the patient was put in supine position and a rigid bronchoscopy is used to extract the tumour which was found occluding the left main bronchus. The procedure was continued uneventfully, and the patient had an uncomplicated postoperative course.

\section{Discussion}

The benefits of using one lung ventilation in VATS have always been established and areee nearly routine practice. The use of DLT for OLV while performing a thoracotomy has not been well studied and is always thought of as an additional benefit to give the surgeon a static field, improve visualization and prevent contralateral spillage but is not an absolute must. Thoracic surgeons who have operated for decades via a thoracotomy without OLV would sometimes choose to keep away from using a DLT particularly with the frequency of unavailability of an experienced anesthetist to insert the tube or a fiberoptic bronchoscopy for proper confirmation tube positioning. Additionally, the potential complications associated with insertion of a DLT are well known. In bronchoplastic procedures for endobronchial tumours, there could be additional anesthetic and surgical concerns for not inserting a double lumen tube and the assumption that this could be safer for patients. This is the first study to explore the anesthetic and surgical benefits of inserting DLT in open thoracotomy cases while performing conventional anatomical lung resections or sleeve resections for endobronchial tumours.

In right side endobronchial tumours, the decision to insert a left sided DLT should be more straightforward. Firstly, right side endobronchial carcinoids (which is the most common endobronchial tumour) are much more common on the right side and secondly, that left side DLT can be performed blindly even with the unavailability of a fiberoptic bronchoscopy [4].

The margin of success in correct placement is higher for placing a left-sided DLT due to the longer length of the corresponding left main stem bronchus. Placement of a right-sided DLT is usually more challenging with a higher chance 
of incorrect placement due to the short length of the right mainstem bronchus and the origination of the right-upper lobe bronchus 1.5 to $2 \mathrm{~cm}$ from the carina [5]. Hence, right-sided DLT incorporates a modified cuff, or an opening on the endobronchial side (called Murphy's eye) that allows ventilation of the right upper lobe. Placement of a right sided DLT needs to be correctly positioned to align with the takeoff of the right upper lobe.

The opponents of placing a double lumen tube in case of a endobronchial lesion requiring a sleeve resection is that the bronchial tube balloon placed just on the carina can interfere with the precise anastomosis of the target lobar bronchus with the main bronchus in cases where the anastomosis must be in close proximity to the carina. We have shown that this is not the case as surgical satisfaction is higher with DLT and even with the highest anastomosis during a bronchoplastic procedure onto the carina, a properly positioned bronchial cuff of a DLT is away from the site of anastomosis. Secondly, there is a concern of inadvertent placement of the bronchial end of the DLT into the wrong main bronchus which can result in traumatic bleeding and soiling of both lungs. These complications are unlikely to occur with a single lumen endo tracheal tube and it is this belief which could have encouraged some surgeons in our series to insist on using an SLT even with the paradoxical self dis-satisfaction with the non-optimal surgical field or due to their previous negative experience with DLT which they felt could breach patient safety.

The concern of significant hemorrhage in the airway after inadvertent intubation of the wrong main bronchus by a double lumen tube is one concern that keeps some thoracic surgeons and anesthetists away from DLT in cases of endoluminal bronchial carcinoid. Carcinoid tumours are vascular in nature and trauma to the tumour can result in significant; occasionally life threatening; hemorrhage. In one review of carcinoid tumors of the lung, tumor biopsy resulted in moderate to severe hemorrhage in 6 out of 23 cases [6]. The endobronchial hemorrhage after bronchoscopic biopsy can be severe enough to warrant the need for lung isolation for contralateral lung protection and avoiding blood spillage which can be potentially fatal. An endobronchial blocker or the rapid proper re-positioning of the DLT in this case can be lifesaving [7]. In fact, in our series 28 out of 106 patients (26\%) referred to surgery had significant bleeding during bronchoscopic biopsy by the chest physician and in some cases, this hindered the availability of preoperative pathological diagnosis. This can explain the concern of surgeons who have been cautioned by the chest physicians of the bleeding nature of the tumour to choose to avoid the DLT to keep away from any potential of contacting the tumour until resection.

Opponents of inserting double lumen tubes during open thoracotomy may be supported by the fact that additional complications may occur with DLTs that can be avoided with SLTs while the procedure is still easily reproducible. The most fatal complication arising from the use of DLTs is airway rupture from inadvertent traumatic placement. Most airway injuries are associated with undersized DLTs, particularly in females who receive a $35 \mathrm{~F}$ or $37 \mathrm{~F}$ tube. Undersized 
DLTs tend to migrate too far distally into the corresponding main stem bronchus, or that the endobronchial cuff requires a larger amount of air when smaller tubes are used predisposing to mucosal ischemia [8]. Other significant complications include hypoxemia due to tube malposition, tube occlusion from blood or secretions, and tube displacement. Inadvertent entrapment of the DLT in suture lines during procedures have also been reported. Malposition of the DLT can lead to life-threatening consequences. Ventilation can be severely impaired, leading to hypoxia, gas trapping, tension pneumothorax, cross-contamination of lung contents, and interference with surgical procedures [9].

In 1992, Russell [10] suggested a simple way for blindly placing a double lumen tube without the need for bronchoscopy. In clinical practice, he relied on his simple way to position a left-sided DLT, in which the inflated bronchial cuff was used as the "carinal hook," and in his preliminary experiments, the method obtained a high success rate. The maneuver relies on partially inflating the bronchial balloon and recreating the effect of a carinal hook on the DLTs to give an idea of orientation and depth. However, Russel did not perform a statistical analysis on the difference between his simple proposed way and the conventional method of insertion.

The alternatives of using double lumen tubes to achieve single lung ventilation can be using bronchial blockers (BB) although the efficacy and simplicity of DLTs are well proven. Bauer and his colleagues [11] have shown that for left side devices, the use of a DLT is preferable to a left BB because of its superior ease of placement. For a right-sided VATS, DLT and right BB showed the same facility of placement but the DLT provided a better quality of lung deflation and a superior surgical view. We believe BB can be more useful to achieve one lung ventilation in the pediatric population where DLT sizes are unavailable. The other alternative is to advance a single lumen tube in the right main bronchus to achieve one lung ventilation after cuff inflation. The disadvantage is that the right upper lobe bronchus is not well ventilated in most cases and there is a higher chance of postoperative right upper lobe collapse.

The correct positioning of double lumen tubes in endobronchial tumour surgery is crucial as wrong positioning has intraoperative anesthetic and surgical implications.

In cases where the tube is too high (not in far enough), the bronchial cuff will herniate into the trachea and when attempting to ventilate the left lung selectively, ventilation of both lungs occurs. When attempting to ventilate the right lung selectively, obstruction to airflow may occur because of the presence of the endobronchial cuff in the distal trachea. In case of a bronchoplastic procedure, the surgeon may struggle with a herniated bronchial cuff in the surgical field if the anastomosis is being performed to the carina.

In cases where the tube is too low (tube in too far), the tip of the endobronchial tube may be beyond the origin of the left upper lobe. When attempting to ventilate the left lung selectively, only the left lower lobe will ventilate. When at- 
tempting to ventilate the right lung selectively, obstruction to airflow occurs because the tracheal lumen is abutting the carina.

The intra procedural migration of an endobronchial tumour has been reported during bronchoscopy [12] but to our knowledge has not been reported in the English literature during surgical resection although it should not be astonishing.

Most endobronchial carcinoids are pedunculated tumours attached by a small stalk to the bronchial wall. Aggressive manipulation during open thoracotomy and manually handling the lung can lead to detachment of the tumour and with airway squeezing and the supine position of the patient, the tumour will migrate proximally to fall in the open contralateral bronchus [13]. The presence of DLT can be highly protective in this setting as the bronchial cuff will seal the contralateral bronchus and if the tumour disconnects from its bronchus and migrates proximally it will theoretically only find the same bronchus again to enter it and hence the independent lung will always be patent.

The importance of the availability of a rigid bronchoscopy as a lifesaving adjunct during the procedure cannot be emphasized more. In all four patients in our series who suffered the catastrophic event, there was a high index of suspicion of the pathophysiology of the event and only the rigid bronchoscopy and its extracting forceps can successfully remove the tumour from the contralateral bronchus within 30 seconds. The fiberoptic bronchoscope forceps do not have the capacity to hold larger tumours. The only alternative to try saving these patients if a rigid bronchoscopy is unavailable is a trial of bronchotomy towards the carinal and inserting forceps to the contralateral bronchus. This does carry a major risk of airway disruption and will frequently be a blind procedure. We would never operate on patients with endobronchial tumours now without the availability of a full rigid bronchoscopy set even with the current routine use of a double lumen tube after the results of this study to achieve maximum safety.

\section{Limitations of the Study}

The non-randomized nature of the study is a limiting factor although it will be difficult to randomize patients to either SLT or DLT where anesthetists/surgeons think that one method is safer for patients and there is no solid evidence in the literature to support either way. We hope a larger randomized study can be initiated in this field.

\section{Conclusion}

We conclude that it is safe to place a double lumen endo tracheal tube for patients with endobronchial tumours requiring open lung resection or bronchoplastic procedures. Moreover, anesthetists should encourage surgeons suggesting not to insert a double lumen tube that avoiding single lung ventilation can be hazardous as the independent lung is exposed to intraoperative tumour migration and bronchial occlusion which can be fatal and the additional 
benefit of increasing surgical satisfaction by reducing: spillage and tidal volume loss during surgical anastomosis of the open airway.

\section{Conflicts of Interest}

The authors declare no conflicts of interest regarding the publication of this paper.

\section{References}

[1] Gaillat, F., Duponq, R., Chabert, L. and Charvet, A. (2011) Editorial Comment: Double-Lumen Tube Intubation Systematic Verification with Fiberoptic Bronchoscopy: Between What Who Should Do and What We Can Do. European Journal of Cardio-Thoracic Surgery, 40, 917-918. https://doi.org/10.1016/j.ejcts.2011.04.006

[2] Miller, R.D., Eriksson, L.I., Fleisher, L.A., Wiener-Kronish, J.P. and Young, W.L. (2010) Anesthesia for Thoracic Surgery. In: Chapter 59 Miller's Anesthesia, 7th Edition, Churchill Livingstone Elsevier, Vol. 2.

[3] Barash, P., Cullen, B.F., Stoelting, R.K., Cahalan, M. and Stock, M.C. (2009) Anesthesia for Thoracic Surgery. In: Chapter 40 Clinical Anesthesia, 6th Edition, Lippincott Williams \& Wilkins.

[4] Zong, Z.J., Shen, Q.Y., Lu, Y. and Li, Y.H. (2016) A Simple Blind Placement of the Left-Sided Double-Lumen Tubes. Medicine, 95, e5376.

https://doi.org/10.1097/MD.0000000000005376

[5] Lewis Jr., J.W., Serwin, J.P., Gabriel, F.S., Bastanfar, M. and Jacobsen, G. (1992) The Utility of a Double-Lumen Tube for One-Lung Ventilation in a Variety of Noncardiac Thoracic Surgical Procedures. Journal of Cardiothoracic and Vascular Anesthesia, 6, 705-710. https://doi.org/10.1016/1053-0770(92)90056-D

[6] Todd, T.R., Cooper, J.D., Weissberg, D., Delarue, N.C. and Pearson, F.G. (1980) Bronchial Carcinoid Tumors: Twenty Years' Experience. The Journal of Thoracic and Cardiovascular Surgery, 79, 532-536. https://doi.org/10.1016/S0022-5223(19)37917-6

[7] Ayache, M., Donatelli, C., Roncin, K., AnsariGilani, K., Yang, M., Faress, J. and Teba, C. (2018) Massive Hemorrhage after Inspection Bronchoscopy for Carcinoid Tumor. Respiratory Medicine Case Reports, 24, 125-128. https://doi.org/10.1016/j.rmcr.2018.05.002

[8] Fitzmaurice, B.G. and Brodsky, J.B. (1999) Airway Rupture from Double-Lumen Tubes. Journal of Cardiothoracic and Vascular Anesthesia, 13, 322-329. https://doi.org/10.1016/s1053-0770(99)90273-2

[9] Luther, D.G.P., Robertson, H.F., Suchett-Kaye, I., Birch, A. and Molyneux, M. (2019) Double-Lumen Tracheal Tubes and Bougies: A Bench Study to Investigate Factors That Influence the Risk of Shearing. Anaesthesia, 74, 891-895. https://doi.org/10.1111/anae.14672

[10] Russell, W.J. (1992) A Blind Guided Technique for Placing Double-Lumen Endobronchial Tubes. Anaesthesia and Intensive Care, 20, 71-74. https://doi.org/10.1177/0310057x9202000114

[11] Bauer, C., Winter, C., Hentz, J.G., Ducrocq, X., Steib, A. and Dupeyron, J.P. (2001) Bronchial Blocker Compared to Doublw-Lumen Tube for One-Lung Ventilation during Thoracoscopy. Acta Anaesthesiologica Scandinavica, 45, 250-254. https://doi.org/10.1111/j.1399-6576.2001.450218.x

[12] Abouzgheib, W., Gaspard, D. and Boujaoude, Z. (2012) Migrating Tumor: A Se- 
rious Complication of Use of Endobronchial Snare Loop. Journal of Bronchology \& Interventional Pulmonology, 19, 262-263.

https://doi.org/10.1097/lbr.0b013e31825cf15e

[13] Gillham, M. (2007) Cardiothoracic Critical Care. Section VI Chapter 40, 573-603. 